\title{
Review
}

Zhe An*

\section{Role of SR-BI in atherosclerosis, malignancies, and infectious diseases}

DOI: $10.2478 / \mathrm{ii}-2018-0011$

Received January 28, 2018; accepted February 06, 2018; published online April 10, 2018

\begin{abstract}
Scavenger receptor class B type I (SR-BI) is a high-affinity receptor for high-density lipoprotein (HDL). The primary role of this receptor is the selective uptake of HDLs in the liver through reverse cholesterol transport. SR-BI interacts with HDL to regulate lipid metabolism and affects various vascular cell functions involved in atherosclerosis (As). In addition, SR-BI is involved in the development of malignant tumors and infectious diseases. This article reviews the function and potential therapeutic targets of SR-BI in As, malignancies, and infectious diseases.
\end{abstract}

Keywords: Scavenger receptor class B, type I; High-density lipoprotein; Atherosclerosis; Malignant neoplasms

Scavenger receptor class B type I (SR-BI) is the first natural membrane receptor for high-density lipoprotein (HDL) at the molecular level. This high-affinity receptor exhibits physiological correlation with HDL. SR-BI is mainly expressed in liver, adrenal, ovarian, and testicular tissues and is also distributed in vascular endothelial cells, smooth muscle cells, macrophages, and adrenal cortical cells. SR-BI is located in the pits on the cell surface and regulates cholesterol in the cell membrane. This protein, with a molecular weight of approximately $82 \mathrm{kDa}$, contains 509 amino acids. SR-BI contains two short intracellular N- and C-terminal cytoplasmic domains, two transmembrane domains, and one large extracellular domain, which consists of 5 to 6 cysteine residues and multiple $\mathrm{N}$-linked glycosylation sites [1,2]. The extracellular loop of SR-BI can interact with many proteins, especially HDL. The predicted sequences of the SR-BI protein in various mammalian species share $70 \%-80 \%$ sequence homology. The classical role of SR-BI is mediating the selective uptake of HDL-cholesterol esters (CE) in vivo and in vitro by reverse cholesterol transport (RCT) [3]. SR-BI also mediates the two-way flow of unesterified cholesterol and phospholipids between lipoproteins and cells [4]. In the liver, it regulates HDL particle size and composition [5]. Numerous studies have shown that SR-BI plays a crucial role in the prevention of atherosclerosis (As). SR-BI is associated with the development of malignancies and infectious diseases. This article summarizes the role of SR-BI and its potential therapeutic targets in As, malignancies, and infectious diseases.

\section{Physiological function of SR-BI}

\subsection{Cell signal transduction}

SR-BI exhibits many physiological functions, including signal function. As a receptor of HDL, SR-BI can directly or indirectly regulate the signal of HDL. Some studies have demonstrated that SR-BI can activate downstream signaling molecules, such as Src kinase, PI3 kinase, Akt kinase, and Erk1/2 mitogen-activated protein kinase. These non-receptor tyrosine kinases form various signaling pathways to stimulate endothelial nitric oxide synthase phosphorylation [6].

Department of Cardiology, China-Japan Union Hospital of Jilin University, Changchun 130033, Jilin, P.R. China

*Correspondence: Zhe An, E-mail: anzhejl@163.com 
SR-BI can initiate multiple signaling pathways after interacting with HDL. The initiation of signaling in endothelial cells requires PDZK1, an adapter protein containing four PDZ domains that recognize the C-terminal domain of SR-BI [7]. The interaction of PDZK1 with SR-BI in the liver significantly affects the metabolism and structure of plasma HDL. PDZK1 is crucial for the activation of many downstream signaling pathways of HDL-mediated Src phosphorylation and SR-BI-mediated HDL stimulation, such pathways include Src activation, eNOS phosphorylation, cell migration, and carotid artery reendothelialization after perivascular electrical injury. Therefore, the interaction of HDL/SR-BI/PDZK1 is essential to maintain the integrity of monolayer endothelial cells. In apoptotic cells and macrophages, SR-BI that binds to phosphatidylserine can mediate Src phosphorylation and plasma membrane accumulation, thereby activating PI3K and Rac1 to engulf and clear apoptotic cells [8]. The lack of SR-BI in macrophages causes defects in the endocytosis signal regulated by Src/PI3K/AKT/Rac1 signaling, resulting in increased plaque area, inflammation, and necrosis.

\subsection{Intracellular cholesterol homeostasis}

SR-BI is the first cell surface receptor that regulates HDL metabolism. In certain types of cells, such as macrophages, adipocytes, and keratinocytes, changes in cholesterol metabolism are associated with the induction of SR-BI expression. The regulation of macrophage cholesterol balance plays a crucial role in the pathogenesis of As, that is, intracellular transport and efflux during cholesterol influx imbalance will lead to the over-accumulation of macrophage cholesterol and conversion to foam cells. Studies have shown that HDL accumulates naturally in atherosclerotic plaques and interacts with ATP-binding cassette transporter A1 (ABCA1), ATP-binding cassette transporter G1 (ABCG1), and SR-BI, causing macrophages with excessive lipids to clear cholesterol through RCT and transport it to the liver [9].

The complex network of programs that triggers cholesterol homeostasis occurs predominantly in the liver. Therefore, the liver is considered a "power station" of metabolism. Dietary cholesterol is transported from the intestine to the liver through HDLs, and all HDL particles are internalized in the liver after interacting with hepatocyte SR-BI. Mature HDL transports cholesterol to the liver directly via SR-BI or indirectly via CETP into apolipoprotein apoB, which is subsequently taken up by the liver via low-density lipoprotein receptor [10]. Therefore, SR-BI plays a key role in maintaining cellular and systemic cholesterol balance. Liver X receptor $\alpha$ $(\mathrm{LXR} \alpha)$ is the target gene of PPAR $\gamma$. The clearance of THP-1 macrophage cholesterol can be increased by the LXR $\alpha / P P A R \gamma$ pathway. The activation of LXR induces the synthesis of polyunsaturated fatty acids (PUFA) in macrophages, leading to significant changes in the lipid components of macrophages. LXR agonist treatment in atherosclerotic lesions regulates the metabolism of PUFAs. Thus, cholesterol transporters (ABCA1, ABCG1, and SR-BI) and nuclear receptors (PPAR $\gamma$ and $L X R \alpha$ ) play an important role in maintaining cholesterol metabolism and homeostasis.

\section{SR-BI-related diseases}

\subsection{SR-BI and As}

As a major cause of cardiovascular diseases, As is not only a state of lipid disorder but also a chronic inflammatory disease characterized by excessive accumulation of cholesterol in the intima of the artery. Mouse models demonstrate that the overexpression of SR-BI prevents As. Moreover, if SR-BI produces similar physiological and pathophysiological effects on humans, then it will be an effective target for treatment and intervention of cardiovascular and reproductive diseases. However, most patients with As lack receptor or carrier deficiencies. The apoA-I (HDL)/SR-BI/eNOS axis is highly protective against deep venous thrombosis (DVT) in the lower extremities and may provide a new target for prevention and treatment of venous 
thrombosis. Experiments directly or indirectly indicate that SR-BI, as a functional receptor for HDL and a potential receptor for identifying additional HDL, may provide new therapeutic avenues for the prevention and/or treatment of As. In addition, organ-targeted SR-BI gene therapy may be an effective approach for the treatment of As because liver and vascular SR-BIs are important in the expression of RCT.

Some studies have shown that using transgenic and knockout mice eliminated the effect of SR-BI on As and have proved the anti-atherosclerotic effect of SR-BI. Treatment of ApoE knockout mice with PPARg or PPARa ligands significantly increased the expression of SR-BI in atherosclerotic lesions. In the absence of lipid load, SR-BI significantly contributed to the bidirectional flow of macrophage cholesterol. SR-BI may play an important role in the regulation and function of cholesterol in macrophages and thus promote its anti-atherosclerotic effect. The effect of SR-BI on HDL cholesterol metabolism and As prevention is indirect and crucial for maintaining normal platelet function and preventing thrombosis. Under the condition of high-fat diet, SR-BI in macrophages can greatly promote the homeostasis of macrophage cholesterol in vivo and is the key to reduce the risk of As.

\subsection{SR-BI and malignant tumors}

Malignant tumors pose a serious threat to human health, and their incidence is increasing annually. Tumor cells are characterized by high levels of CEs and low tumor-associated HDLs. Studies have verified the complex relationship between HDL and malignant tumors and elucidated the potential role of SR-BI, a high-affinity receptor for HDL, in the development of malignant tumors. Animal experiments found that in the SR-BI knockout mouse model, the tumor area as well as in vitro cell proliferation and migration decreased. Lu et al. [11] constructed recombinant HDL (rHDL)/MB/CCPs on the basis of rHDL to develop a safe tumor-targeting probe for tumor detection. SR-BI is highly expressed in multiple tumor cell lines, including prostate, breast, liver, rectal, pancreatic, ovarian, and nasopharyngeal cells. The role of HDL/SR-BI in prostate and breast cancers has been the most extensively studied. The expression level of SR-BI is associated with invasiveness and low survival rate in breast and prostate cancers [12].

Prostate cancer is one of the most common solid organ malignancies in men. The presence of a number of unfavorable factors, such as high-fat high-cholesterol diet and hyperlipidemia, can increase the risk of prostate cancer [13]. SR-BI plays a key role in the incidence and development of prostate cancer, especially in the secretion and activity of antigens in prostate cancer cells. Downregulation of SR-BI in prostate cancer cells (C4-2 and LNCaP cell lines) significantly reduces the viability of cells and the secretion of prostatespecific antigen (PSA) [14]. In addition, SR-BI is a key protein of cholesterol influx in prostate cancer cells, and its expression increases during the progression to castration-resistant prostate cancer [15]. Although SRBI activates cholesterol synthesis in C4-2 cells, it still has a significant effect on the production of PSA and the viability of cap (CaP) cells. Hence, further studies must explore SR-BI in human malignancies and determine whether it can be used as a target for the treatment of castration-resistant diseases.

Breast cancer is a major public health problem for women worldwide and has a high incidence in western countries. Epidemiological studies have shown that certain Western lifestyles, such as dietary fat intake, obesity, and alcohol consumption, may increase the risk of breast cancer. Analysis of serum lipoprotein levels in patients with cancer suggests that HDL levels are mainly affected by tumor progression [15]. The physiological receptor SR-BI of HDL is associated with the development of breast cancer. However, the clinical significance of this receptor in breast cancer remains unclear. HDL, as a growth factor in breast cancer, can transport cholesterol to cells via SR-BI. Downregulation of SR-BI in breast cancer cell lines is associated with decreased intracellular cholesterol levels and reduced tumor invasiveness. However, the uncoupling of SR-BI-regulated signaling pathway with cholesterol homeostasis remains to be determined. Highly expressed SR-BI is associated with traditional parameters of increasing types of malignancies and thus may be a new marker for prognosis and incomplete clinical treatment outcome of human breast cancer. 


\subsection{SR-BI and infectious diseases}

SR-BI plays an important role in infectious diseases, especially sepsis and hepatitis C virus (HCV). SR-BI knockout mice displayed extreme phenotypes of endotoxic shock and sepsis. Gilibert et al. [16] demonstrated that the main role of SR-BI in host defense against endotoxic shock is related to its expression in the adrenal cortex. After endotoxic shock or bacterial infection, adrenal SR-BI, a key component of the hypothalamicpituitary-adrenal axis, effectively provides glucocorticoid-dependent host defense. Hepatitis B virus and HCV infection remain a global health problem and cause approximately 450-500 million chronic infections worldwide [17]. Many infections are asymptomatic, and persistent infections are present in $60 \%-80 \%$ of such cases. Chronic infection can lead to severe liver fibrosis, cirrhosis, liver failure, and hepatocellular carcinoma and is the second leading cause of cancer-related deaths [17]. HCV is not only an enveloped virus but also a human pathogen that belongs to the Hepatitis genus of the Flaviviridae family. HCV has two envelope glycoproteins, namely, E1 and E2. E2, which is important for viral entry, binds to host cytokines SR-BI and CD81 and evades host immune responses. SR-BI, human CD81, occludin (OCLN), and claudin 1 (CLDN1) are key receptors or co-receptors for HCV entry into cells; meanwhile, SR-BI and CD81 in treeshrews may interact with HCV envelope protein 2 (E2).

The two natural ligands for SR-BI, namely, HDL and ox-LDL, may promote and inhibit the entry of HCV, respectively. Ox-LDL inhibits the interaction between HCV and SR-BI in a noncompetitive manner. Philipp Solbach et al. [18] demonstrated that ox-LDL is not an inflammatory marker or an interferon response regulator but a potent inhibitor of the proliferation of HCV from cell to cell. Scholars have produced a human SR-BI monoclonal antibody (mAb), namely, mAb16-71 to effectively inhibit Huh-7.5 liver cancer cell infection, primarily hepatocyte culture-derived HCVcc; the co-receptor SR-BI of the human mAbs targeting HCV can completely inhibit the infection and spread of different HCV genotypes in the liver [19]. SRBI mAbs, mainly mAb8 and mAb151, can be developed as new therapeutic approaches for inhibiting HCV reinfection following liver transplantation. Zuiani et al. [20] found that all virus attachments are similar to those edited by the SR-BI gene. The interaction among the attachments after the mutated viral infection requires the participation of SR-BI. Therefore, studies have used large-scale mutagenesis to identify the variants of the virus. SR-BI was identified as a potential receptor for HCV based on the interaction of its extracellular loop with the envelope glycoprotein E2. After binding, SR-BI initiates and maintains HCV infection and does not require receptor-E2/ HDL interaction. Studies have confirmed that the key receptor for targeting host cells is SR-BI, which mediates the infection and spread of the virus in the liver. Human SR-BI is a key receptor for HCV and is involved in the early stages of HCV infection. SR-BI, which reacts with the HCV envelope, may be a species-specific protein component. SR-BI can prevent graft infection in patients with chronic HCV infection during normal liver transplantation and suppress the presence of HCV escape mutants and virus rebounds during or after antiviral treatment. Therefore, SR-BI targets may represent a new immunotherapy strategy.

\section{Summary and Outlook}

SR-BI can prevent As, regulate HDL metabolism, treat DVT, function as a potential therapeutic target for various malignancies, and inhibit HCV infection. Moreover, the elucidation of the mechanisms of SR-BI function and intermolecular interactions has helped develop effective SR-BI-based drug delivery strategies. Although the primary function of SR-BI has been discovered for over 20 years, its physiological significance remains controversial. Lipid-lowering drugs have been successfully used in the past 26 years, and new therapeutic strategies for vascular wall inflammation and markers of As have been only developed in recent years [9]. The distribution of blood lipids, especially HDL and LDL cholesterol, is an important tool for monitoring the risk of As. However, whether or not SR-BI-mediated efflux originates from a different or similar cell pool of cholesterol remains unclear. The function of SR-BI and its therapeutic potential in diseases, including As, malignancies, and infections, should be further studied. 
Acknowledgments: None.

Conflict of interest: The author states no conflicts of interest.

\section{References}

[1] Vaisman B.L., Vishnyakova T.G., Freeman L.A., Amar M.J., Demosky S.J., Liu C., et al., Endothelial expression of scavenger receptor class B, type I protects against development of atherosclerosis in mice, Biomed. Res. Int., 2015, 2015, 607120, DOI: $10.1155 / 2015 / 607120$

[2] Shen W.J., Hu J., Hu Z., Kraemer F.B., Azhar S., Scavenger receptor class B type I (SR-BI): a versatile receptor with multiple functions and actions, Metab. Clin. Exp., 2014, 63, 875-886, DOI: 10.1016/j.metabol.2014.03.011

[3] Trigatti B.L., Fuller M., HDL signaling and protection against coronary artery atherosclerosis in mice, J Biomed. Res., 2015, 30, 94-100

[4] Mo Z.C., Ren K., Liu X., Tang Z.L., Yi G.H., A high-density lipoprotein-mediated drug delivery system, Adv. Drug Deliv. Rev., 2016, 106(Pt A), 132-147, DOI: 10.1016/j.addr.2016.04.030

[5] Chadwick A.C., Sahoo D., Functional genomics of the human high-density lipoprotein receptor scavenger receptor BI: an old dog with new tricks, Curr. Opin. Endocrinol. Diabetes Obe., 2013, 20, 124-131, DOI: 10.1097/MED.0b013e32835ed575

[6] Saddar S., Carriere V., Lee W.R., Tanigaki K., Yuhanna I.S., Parathath S., et al., Scavenger receptor class B type I is a plasma membrane cholesterol sensor, Circ. Res., 2013, 112, 140-151, DOI: 10.1161/CIRCRESAHA.112.280081

[7] Zannis V.I., Fotakis P., Koukos G., Kardassis D., Ehnholm C., Jauhiainen M., et al., HDL biogenesis, remodeling, and catabolism, Handb. Exp. Pharmacol., 2015, 224, 53-111, DOI: 10.1007/978-3-319-09665-0_2

[8] Tao H., Yancey P.G., Babaev V.R., Blakemore J.L., Zhang Y., Ding L., et al., Macrophage SR-BI mediates efferocytosis via Src/PI3K/Rac1 signaling and reduces atherosclerotic lesion necrosis, J. Lipid Res., 2015, 56, 1449-1460, DOI: $10.1194 /$ jlr.M056689

[9] Sanchez-Gaytan B.L., Fay F., Lobatto M.E., Tang J., Ouimet M., Kim Y., et al., HDLmimetic PLGA nanoparticle to target atherosclerosis plaque macrophages, Bioconjug. Chem., 2015, 26, 443-451, DOI: 10.1021/bc500517k

[10] Hu Y.W., Ma X., Huang J.L., Mao X.-R., Yang J.-Y., Zhao J.-Y., et al., Dihydrocapsaicin attenuates plaque formation through a PPAR gamma/LXR alpha pathway in apoE mice fed a high-fat/high-cholesterol diet, PLoS ONE, 2013, 8:e66876, DOI: $10.1371 /$ journal.pone.0066876

[11] Lu H., Zhang H., Zhang D., Lu H., Ma D., A biocompatible reconstituted high-density lipoprotein nano-system as a probe for lung cancer detection, Med. Sci. Monit., 2015, 21, 2726-2733, DOI: 10.12659/MSM.895255

[12] Mooberry L.K., Sabnis N.A., Panchoo M., Nagarajan B., Lacko A.G., Targeting the SR-B1 receptor as a gateway for cancer therapy and imaging, Front. Pharmacol., 2016, 7:466, DOI: 10.3389/fphar.2016.00466

[13] Schorghofer D., Kinslechner K., Preitschopf A., Schütz B., Röhrl C., Hengstschläger M., et al., The HDL receptor SR-BI is associated with human prostate cancer progression and plays a possible role in establishing androgen independence, Reprod. Biol. Endocrinol., 2015, 13, 88, DOI: 10.1186/s12958-015-0087-z

[14] Gutierrez-Pajares J.L., Ben Hassen C., Chevalier S., Frank P.G., SR-BI: linking cholesterol and lipoprotein metabolism with breast and prostate cancer, Front. Pharmacol., 2016, 7:338, DOI: 10.3389/fphar.2016.00338

[15] Yuan B., Wu C., Wang X., Wang D., Liu H., Guo L., et al., High scavenger receptor class B type I expression is related to tumor aggressiveness and poor prognosis in breast cancer, Tumour Biol., 2016, 37, 3581-3588, DOI: $10.1007 / \mathrm{s} 13277-015-4141-4$

[16] Gilibert S., Galle-Treger L., Moreau M., Saint-Charles F., Costa S., Ballaire R., et al., Adrenocortical scavenger receptor class B type I deficiency exacerbates endotoxic shock and precipitates sepsis-induced mortality in mice, J. Immunol., 2014, 193, 817-826, DOI: 10.4049/jimmunol.1303164

[17] Colpitts C.C., Verrier E.R., Baumert T.F., Targeting viral entry for treatment of hepatitis B and C virus infections, ACS Infect. Dis., 2015, 1, 420-427, DOI: 10.1021/acsinfecdis.5b00039

[18] Solbach P., Westhaus S., Deest M., Herrmann E., Berg T., Manns M.P., et al., Oxidized low-density lipoprotein is a novel predictor of interferon responsiveness in chronic hepatitis C infection, Cell. Mol. Gastroenterol. Hepatol., 2015, 1, 285-294, DOI: 10.1016/j.jcmgh.2015.03.002

[19] Meuleman P., Catanese M.T., Verhoye L., Desombere I., Farhoudi A., Jones C.T., et al., A human monoclonal antibody targeting scavenger receptor class B type I precludes hepatitis $C$ virus infection and viral spread in vitro and in vivo, Hepatology, 2012, 55, 364-372, DOI: 10.1002/hep.24692

[20] Zuiani A., Chen K., Schwarz M.C., White J.P., Luca V.C., Fremont D.H., et al., A library of infectious hepatitis C viruses with engineered mutations in the $\mathrm{E} 2$ gene reveals growth-adaptive mutations that modulate interactions with scavenger receptor class B type I, J. Virol., 2016, 90, 10499-10512, DOI: 10.1128/JVI.01011-16 